\title{
New earthworm records from various parts of Greece (Oligochaeta: Lumbricidae, Acanthodrilidae, Megascolecidae, Ocnerodrilidae)
}

\author{
T. SZEDERJESI
}

\begin{abstract}
Tímea Szederjesi, Department of Systematic Zoology and Ecology, Eötvös Loránd University, H-1117 Budapest, Pázmány P. sétány 1/C. E-mail: t.szederjesi@gmail.com
\end{abstract}

\begin{abstract}
Elaboration of new earthworm materials collected from the continental and insular parts of Greece resulted in recording altogether 22 species and subspecies, of which three, namely Pontodrilus litoralis (Grube, 1855), Ocnerodrilus occidentalis Eisen, 1878 and Dendrobaena pentheri (Rosa, 1905) as senior synonym of Dendrobaena aegea (Cognetti, 1913) proved to be new to the fauna of Greece. Consequently, the number of the earthworm species recorded for Greece is raised to 59.
\end{abstract}

Keywords. Earthworms, faunistics, new records, Greece.

\section{INTRODUCTION}

$\mathrm{T}$ he earthworm fauna of continental Greece is quite well-known. After Michaelsen (1902, 1914), several other scientists carried out researches here and published new data from the country (Tzelepis 1943, Omodeo 1955, Karaman 1972, Šapkarev 1972, Michalis 1975a, 1976, 1977, Zicsi 1973, 1974). Finally, Zicsi \& Michalis (1981) summarized the knowledge on the lumbricid earthworms of Greece. After this comprehensive work, four new species and many new data were added to the earthworm fauna of Greece (Michalis 1982, 1983, 1987, 1995, Zicsi \& Michalis 1993, Szederjesi \& Csuzdi 2012).

The situation is just the opposite regarding the Greek islands, from where we have only sporadic data. Only five papers dealt with the insular earthworm fauna; Cognetti (1913) described two new species, Perelia phoebea and Dendrobaena aegea from Rhodes, and published new data from the island. Michaelsen (1928) described Eisenia ariadne from Naxos and recorded the presence of Octodrilus complanatus (Dugès, 1828) and Lumbricus rubellus Hoffmeister, 1843 from Lemnos. Cognetti (1906) and Černosvitov (1934) published new data from Crete and Michalis (1975b) from the island Kos.
Besides the 54 recorded earthworm species from the family Lumbricidae (Szederjesi \& Csuzdi 2012), two species, namely Microscolex dubius (Fletcher, 1887) and M. phosphoreus Dugès, 1837 from the family Acanthodrilidae (Michalis 1975a, 1975b, 1987, Szederjesi \& Csuzdi 2012) and two species, Pheretima heterochaeta (Michaelsen, 1891) and Ph. peregrina $\mathrm{v}$. papillosa (Tzelepis, 1943) from the family Megascolecidae (Michalis 1976, 1982) are also recorded from Greece. To our present knowledge, these two Pheretima species are synonyms of Amynthas corticis (Kinberg, 1867) (Csuzdi 2012).

In the last decade, researchers of the Hungarian Natural History Museum organized several collecting trips to Greece, including continental and insular regions too. This present paper summarizes the results of the collecting expeditions to the Pindus mountain range, Thrace, Rhodes, Karpathos, Crete, and Naxos between 2012 and 2014.

\section{MATERIAL AND METHODS}

Earthworms were collected by the diluted formaldehyde method (Raw 1959), complemented with digging and searching under stones and under the bark of fallen logs. The specimens were killed and fixed in $96 \%$ ethanol, then transferred 
into $75 \%$ ethanol and deposited in the earthworm collection of the Hungarian Natural History Museum (HNHM). For later molecular studies, some specimens were placed into $96 \%$ ethanol.

\section{RESULTS}

\section{Family Lumbricidae Rafinesque-Schmaltz, 1815}

\section{Aporrectodea caliginosa (Savigny, 1826)}

Enterion caliginosum, Savigny 1826: 180.

Allolobophora caliginosa: Zicsi \& Michalis 1981: 247., 1993: 302., Michalis 1982: 350., 1987: 61., 1995: 15.

Aporrectodea caliginosa: Szederjesi \& Csuzdi 2012: 29.

Material examined. HNHM/16176 2 ex., South Aegean, Rhodes regional unit, Vati, roadside spring $E$ of the village, $75 \mathrm{~m}, \mathrm{~N} 36^{\circ} 03.225^{\prime}$ E2754.486', leg. J. Kontschán, D. Murányi, 08. 11.2012. HNHM/16188 1 ex., South Aegean, Rhodes regional unit, Afandou, olive groove $\mathrm{N}$ of the village, $105 \mathrm{~m}, \mathrm{~N} 36^{\circ} 18.167^{\prime}$ E28 08.964 ', leg. J. Kontschán, D. Murányi, 13.11.1012. HNHM/16627 1 ex., South Aegean, Naxos regional unit, Mt. Zas, Filoti, vicinity of Zas Cave, 680 m, N3701.9' E2529.8', leg. J. Kontschán, D. Murányi, T. Szederjesi, 07.04.2013. HNHM/ 166331 ex., Crete, Rethymno regional unit, Ida Mts., spring and its outlet at an archeogical site and taverna, nest of ants, $1380 \mathrm{~m}, \mathrm{~N} 35^{\circ} 12.388^{\prime}$ E2450.044', leg. J. Kontschán, D. Murányi, T. Szederjesi, 02.04.2013. HNHM/16639 1 ex., Crete, Lasithi regional unit, Zakros, Zakros Spring above the village, wet litter of plane tree, 265 m, N35 $06.837^{\prime}$ E26 ${ }^{\circ} 12.827^{\prime}$, leg. J. Kontschán, D. Murányi, T. Szederjesi, 04.04.2013. HNHM/16641 1 ex., Crete, Heraklion regional unit, Loutraki, stream and its gorge below the village, plane tree litter, $670 \mathrm{~m}, \mathrm{~N} 35^{\circ} 03.413^{\prime}$ E2524.887', leg. J. Kontschán, D. Murányi, T. Szederjesi, 05.04.2013. HNHM/16647 1 ex., Crete, Lasithi regional unit, Thriptis Mts, Orino, stream in the village, $625 \mathrm{~m}, \mathrm{~N} 35^{\circ} 04.883^{\prime}$ E2554.848', leg. J. Kontschán, D. Murányi, T.
Szederjesi, 05.04.2013. HNHM/16658 1 ex., Crete, Lasithi regional unit, Agios Georgios, large reservoir below the village, $60 \mathrm{~m}, \mathrm{~N} 35^{\circ} 03.042^{\prime}$ E2541.750', leg. J. Kontschán, D. Murányi, T. Szederjesi, 05.04.2013. HNHM/16666 1 ex., Crete, Chania regional unit, Lefka Ori Mts, Omalos, rocky grassland $\mathrm{W}$ of the village, $1060 \mathrm{~m}$, N35¹9.483' E2353.507', leg. J. Kontschán, D. Murányi, T. Szederjesi, 31.03.2013.

\section{Aporrectodea jassyensis (Michaelsen, 1891)}

Allolobophora jassyensis Michaelsen, 1891: 15., Michalis 1987: 62.

Allolobophora jassyensis jassyensis: Zicsi \& Michalis 1981: 250., 1993: 303. Michalis 1982: 352., Michalis et al. 1989: 5.

Aporrectodea jassyensis: Szederjesi \& Csuzdi 2012: 30.

Material examined. HNHM/16075 1 ex., Thrace, Rhodope peripheral unit, Sapka Mts., Kizario, stream and pasture $\mathrm{SW}$ of the village, $140 \mathrm{~m}$, N41 ${ }^{\circ} 03.492^{\prime}$ E2545.672', leg. J. Kontschán, D. Murányi, T. Szederjesi, 27.05.2012. HNHM/ 166402 ex., South Aegean, Naxos regional unit, Dhamalas, rocky phrygana $\mathrm{W}$ of the village, 260 m, N3702.921’ E2527.351', leg. J. Kontschán, D. Murányi, T. Szederjesi, 07.04.2013. HNHM/ 166441 ex., Crete, Lasithi regional unit, Karidi, rocky grassland $\mathrm{W}$ of the village, $290 \mathrm{~m}$, N3507.912' E26² 12.849', leg. J. Kontschán, D. Murányi, T. Szederjesi, 04.04.2013.

\section{Aporrectodea rosea (Savigny, 1826)}

Enterion roseum Savigny, 1826: 182.

Allolobophora rosea: Zicsi \& Michalis 1981: 249., Michalis 1975: 204., 1976: 156., 1977: 286., 1982: 350., 1987: 62.

Allolobophora rosea f. typica: Michalis 1975: 189. Allolobophora rosea bimastoides: Michalis 1982: 350.

Material examined. HNHM/16626 1 ex., South Aegean, Naxos regional unit, Mt. Zas, Filoti, vicinity of Zas Cave, $680 \mathrm{~m}$, N37 $01.9^{\prime}$ E2529.8', leg. J. Kontschán, D. Murányi, T. Szederjesi, 07.04.2013. HNHM/16637 2 ex., South Aegean, Naxos regional unit, Ghalini, open 
stream at the village, $35 \mathrm{~m}, \quad \mathrm{~N} 37^{\circ} 06.888^{\prime}$ E25²5.715', leg. J. Kontschán, D. Murányi, T. Szederjesi, 06.04.2013.

\section{Dendrobaena attemsi (Michaelsen, 1902)}

Helodrilus (Dendrobaena) attemsi Michaelsen, 1902: 74.

Dendrobaena attemsi: Zicsi \& Michalis 1981: 258., Michalis 1982: 356., Szederjesi \& Csuzdi 2012: 31.

Material examined. HNMH/16657 3 ex., South Aegean, Naxos regional unit, Koronidha, streamin a gorge below the village, wet soil, 455 m, N37º $08.580^{\prime}$ E $25^{\circ} 31.857^{\prime}$, leg. J. Kontschán, D. Murányi, T. Szederjesi, 06.04.2013. HNHM/ 169461 ex., Thessaly, Trikala peripheral unit, Lakmos Mts., Chaliki, stream below Verliga Waterfall, $1935 \mathrm{~m}, \mathrm{~N} 39^{\circ} 40.674^{\prime} \mathrm{E} 21^{\circ} 07.818$ ', leg. T. Kovács, D. Murányi, 09.05.2014.

\section{Dendrobaena byblica byblica (Rosa, 1893)}

Allolobophora byblica Rosa, 1893: 4-5.

Dendrobaena byblica: Zicsi \& Michalis 1981: 259., Michalis 1982: 356., Michalis et al. 1989: 5.

Eiseniella oltenica: Zicsi \& Michalis 1981: 243.

Dendrobaena byblica byblica: Szederjesi \& Csuzdi 2012: 31 .

Material examined. HNHM/16178 1 ex., South Aegean, Rhodes regional unit, Platania, 'Koinotis Platania' Spring W of the village, 285 m, N36 $15.321^{\prime}$ E28 $00.129^{\prime}$, leg. J Kontschán, D. Murányi. 09.11.2012. HNHM/16185 1 ex., South Aegean, Karpathos regional unit, Spoa, Plakakia, open spring along the road, $215 \mathrm{~m}$, N35 $39.380^{\prime}$ E2709.474', leg. J. Kontschán, d. Murányi, 11.11.2012. HNHM/16187 1 ex., South Aegean, Rhodes regional unit, Petaloudes, 'Butterfly Valley', stream gorge, $190 \mathrm{~m}, \mathrm{~N} 36^{\circ}$ 20.269' E2803.716', leg. J. Kotschán, D. Murányi, 13.11.2012. HNHM/16620 1 ex., Crete, Heraklion regional unit, Agii Deka, small river and its gallery at Agios Tilos basilica, $160 \mathrm{~m}$, N3503.704' E245 56.792', leg. J. Kontschán, D. Murányi, T. Szederjesi, 02.04.2013. HNHM/ 166223 ex., Crete, Lasithi regional unit, Thriptis Mts, Agios Ioannis, stream and its gallery $\mathrm{E}$ of the village, $460 \mathrm{~m}, \mathrm{~N} 35^{\circ} 03.615^{\prime} \mathrm{E} 25^{\circ} 51.938^{\prime}$, leg. J. Kontschán, D. Murányi, T. Szederjesi, 05.04. 2013. HNHM/16651 7 ex., Crete, Rethymno regional unit, Moni Veni, springs at the monastery, soil, $595 \mathrm{~m}, \quad \mathrm{~N} 35^{\circ} 16.228^{\prime}$ E24 ${ }^{\circ}$ 36.377', leg. J. Kontschán, D. Murányi, T. Szederjesi, 01.04.2013. HNHM/16656 2 ex., South Aegean, Naxos regional unit, Koronidha, streamin a gorge below the village, wet soil, 455 m, N3708.580' E2531.857', leg. J. Kontschán, D. Murányi, T. Szederjesi, 06.04.2013. HNHM/ 166601 ex., Crete, Chania regional unit, Kakopetros, stream and its plane tree gallery near the village, plane tree litter, $430 \mathrm{~m}, \mathrm{~N} 35^{\circ} 24.803^{\prime}$ ' E2345.391', leg. J. Kontschán, D. Murányi, T. Szederjesi, 31.03.2013. HNHM/16661 4 ex., Crete, Lasithi regional unit, Zakros, stream and its plane tree gallery $\mathrm{N}$ of the village, mixed soil and leaf litter, $190 \mathrm{~m}, \mathrm{~N} 35^{\circ} 06.918^{\prime}$ E26 ${ }^{\circ} 13.153$ ', leg. J. Kontschán, D. Murányi, T. Szederjesi, 04. 04.2013. HNHM/16662 4 ex., Crete, Rethymno regional unit, Mirthios, D. Dason Rethymnis spring E of the village, $155 \mathrm{~m}, \mathrm{~N} 35^{\circ} 17.619^{\prime} \mathrm{E} 24^{\circ}$ 33.360', leg. J. Kontschán, D. Murányi, T. Szederjesi, 01.04.2013.

\section{Dendrobaena byblica olympiaca (Michaelsen, 1902)}

Dendrobaena ganglbaueri olympiaca Michaelsen, 1902: 47.

Debdrobaena byblica: Zicsi \& Michalis 1981: 259 (part.)

Dendrobaena byblica olympiaca: Szederjesi \& Csuzdi 2012: 32.

Material examined. HNHM/16625 2 ex., South Aegean, Naxos regional unit, Mt. Zas, Filoti, vicinity of Zas Cave, $680 \mathrm{~m}, \mathrm{~N} 37^{\circ} 01.9^{\prime}$ E25'29.8', leg. J. Kontschán, D. Murányi, T. Szederjesi, 07.04.2013. HNHM/16629 4 ex., South Aegean, Naxos regional unit, Apiranthos, olive orchard E of the village, $525 \mathrm{~m}$, N3704.408' E2531.842', leg. J. Kontschán, D. Murányi, T. Szederjesi, 07.04.2013. HNHM/ 166492 ex., South Aegean, Naxos regional unit, Koronidha, stream above the village, $670 \mathrm{~m}$, N3708.071' E2531.507', leg. J. Kontschán, D. Murányi, T. Szederjesi, 06.04.2013. 


\section{Dendrobaena cognettii (Michaelsen, 1903)}

Helodrilus cognettii Michaelsen, 1903: 130.

Dendrobaena cognettii: Zicsi \& Michalis 1981: 260., 1993: 306., Michalis 1982: 356., Szederjesi \& Csuzdi 2012: 32.

Material examined. HNHM/16630 1 ex., South Aegean, Naxos regional unit, Apiranthos, olive orchard $\mathrm{E}$ of the village, $525 \mathrm{~m}$, N37 $04.408^{\prime}$ E25 $31.842^{\prime}$, leg. J. Kontschán, D. Murányi, T. Szederjesi, 07.04.2013.

\section{Dendrobaena hortensis (Michaelsen, 1890)}

Allolobophora subrubicunda var. hortensis Michaelsen, 1890: 15.

Dendrobaena hibernica (Friend, 1892): Michalis 1982: 356.

Dendrobaena hortensis: Michalis 1995: 16., Szederjesi \& Csuzdi 2012: 32.

Material examined. HNHM/16180 1 ex., South Aegean, Rhodes regional unit, Prophitis Ilias, rocky cedar forest at the monastery, $605 \mathrm{~m}$, N36 $16.624^{\prime}$ E2 $7^{\circ} 56.543$ ', leg. J. Kontschán, D. Murányi, 07.11.2012. HNHM/16352 1 ex., South Aegean, Rhodes regional unit, Salakos, 'Butterfly River', a gorge $\mathrm{NE}$ of the city, $135 \mathrm{~m}$, N36 ${ }^{\circ} 17.391$ ' E27 $57.007^{\prime}$, leg. J. Kontschán, D. Murányi, 10.11.2012. HNHM/16632 5 ex., Crete, Rethymno regional unit, Ida Mts., spring and its outlet at an archeogical site and taverna, nest of ants, $1380 \mathrm{~m}, \mathrm{~N}^{\circ} 5^{\circ} 12.388^{\prime}$ E24 ${ }^{\circ} 50.044^{\prime}$, leg. J. Kontschán, D. Murányi, T. Szederjesi, 02.04. 2013. HNHM/16642 2 ex., Crete, Heraklion regional unit, Loutraki, stream and its gorge below the village, plane tree litter, $670 \mathrm{~m}, \mathrm{~N} 35^{\circ} 03.413^{\prime}$ E25 $24.887^{\prime}$, leg. J. Kontschán, D. Murányi, T. Szederjesi, 05.04.2013.

\section{Dendrobaena pentheri (Rosa, 1905)}

Allolobophora (Notogama) pentheri Rosa, 1905: 1. Dendrobaena pentheri: Szederjesi et al. 2014: 560.

Helodrilus (Dendrobaena) aegeus: Cognetti 1913: 4. Syn. nov.

Dendrobaena aegea: Zicsi \& Michalis 1981: 260.
Material examined. HNHM/16179 1 ex., South Aegean, Rhodes regional unit, Prophitis Ilias, rocky cedar forest at the monastery, $605 \mathrm{~m}$, N36² 16.624' E2756.543', leg. J. Kontschán, D. Murányi, 07.11.2012. HNHM/16181 3 ex., South Aegean, Rhodes regional unit, Aghios Nektarios, pine forest $\mathrm{E}$ of the monastery, $145 \mathrm{~m}, \mathrm{~N} 36^{\circ}$ 15.943' E2804.822', leg. J. Kontschán, D. Murányi, 14.11.2012. HNHM/16184 3 ex., South Aegean, Rhodes regional unit, Mt. Atavyros peak region, rocky grassland, $1095 \mathrm{~m}, \mathrm{~N} 36^{\circ} 12.233^{\prime}$ E2751.913', leg. J. Kontschán, D. Murányi, 07.11.2012. HNHM/16186 1 ex., South Aegean, Karpathos regional unit, Lefkos, pine forest $\mathrm{S}$ of the village, $135 \mathrm{~m}, \mathrm{~N} 35^{\circ} 35.730^{\prime} \mathrm{E} 27^{\circ} 05.577^{\prime}$, leg. J. Kontschán, D. Murányi, 12.11.2012.

Remarks. Cognetti (1913) described Dendrobaena aegea from Rhodes. According to the original description, this species is very similar to $D$. pentheri; the slight differences are in the position of the tubercles (29-31 vs. $1 / 228-1 / 232)$ and the opening of the spermathecal pores $(d v s$. $d-\mathrm{M})$.

Zicsi \& Michalis (1981) also recorded D. aegea from Rhodes and mentioned that their specimens differ from the type material in the length of the tubercula pubertatis which is on $1 / 2$ $28-1 / 232$.

The Dendrobaena aegea specimens (HNHM/ 6449) reported by Zicsi \& Michalis (1981) and two syntype specimens (HNHM/12675), and also D. pentheri specimens from Turkey (HNHM/ 16583, 16604) and Cyprus (HNHM/1 4664) were re-examined to clear this question.

All the examined specimens' tubercles show very similar extension, stretching from $1 / n 28$ to $1 / n$ 32, the $D$. aegea syntypes examined have tubercles on $29-1 / 232$. The position of the spermathecal pores show geographic transition. The spermathecae of the specimens from Rhodes open in setal line $d$, from Turkey in setal line $d$ or just above $d$, and from Cyprus the specimens has openings between $d$ and the mid-dorsal line, or 
near the mid-dorsal line. Besides, all specimens have tanylobic head.

Considering all these facts $D$. aegea is proposed as a synonym of Dendrobaena pentheri and in this sense $D$. pentheri is a new species name in the list of the earthworm fauna of Greece.

\section{Dendrobaena veneta (Rosa, 1886)}

Allolobophora veneta Rosa, 1886: 674.

Dendrobaena veneta: Zicsi \& Michalis 1981: 258., Michalis 1982: 355., 1995: 16.

Dendrobaena veneta veneta: Szederjesi \& Csuzdi 2012: 34 .

Material examined. HNHM/16189 6 ex., South Aegean, Rhodes regional unit, Afandou, olive groove $\mathrm{N}$ of the village, $105 \mathrm{~m}$, N36 ${ }^{\circ} 18.167^{\prime}$ E28 $08.964^{\prime}$, leg. J. Kontschán, D. Murányi, 13.11.1012. HNHM/16619 1 ex., Crete, Heraklion regional unit, Agii Deka, small river and its gallery at Agios Tilos basilica, $160 \mathrm{~m}$, N3503.704' E2456.792', leg. J. Kontschán, D. Murányi, T. Szederjesi, 02.04.2013. HNHM/ 166311 ex., Crete, Rethymno regional unit, Ida Mts., spring and its outlet at an archeogical site and taverna, nest of ants, $1380 \mathrm{~m}, \mathrm{~N} 35^{\circ} 12.388^{\prime}$ E2450.044', leg. J. Kontschán, D. Murányi, T. Szederjesi, 02.04.2013. HNHM/16635 2 ex., Crete, Chania regional unit, Lefka Ori Mts, Samaria, spring in oak stand at the rest area, 1250 m, N35 ${ }^{\circ} 18.481^{\prime}$ E235 55.051 ', leg. J. Kontschán, D. Murányi, T. Szederjesi, 31.03.2013. HNHM/ 166431 ex., Crete, Lasithi regional unit, Dikti Mts., Pinakiano, slow stream in a meadow, $\mathrm{W}$ of the village, $815 \mathrm{~m}, \mathrm{~N} 35^{\circ} 11.615^{\prime} \mathrm{E} 25^{\circ} 25.976$ ', leg. J. Kontschán, D. Murányi, T. Szederjesi, 03.04.2013. HNHM/16648 2 ex., Crete, Rethymno regional unit, Ida Mts., limestone rocks at a pasture towards the observatory, $1480 \mathrm{~m}, \mathrm{~N} 35^{\circ}$ 12.560' E2452.536', leg. J. Kontschán, D. Murányi, T. Szederjesi, 02.04.2013.

\section{Dendrodrilus rubidus subrubicundus (Eisen, 1873)}

Allolobophora subrubicunda Eisen, 1873: 51.

Dendrobaena rubida subrubicunda: Zicsi \& Michalis 1981: 257., Michalis 1982: 355., 1987: 63.
Dendrodrilus rubidus subrubicundus: Szederjesi \& Csuzdi 2012: 35.

Material examined. HNHM/16183 1 ex., South Aegean, Karpathos regional unit, Aperi, spring and stream in the village, $265 \mathrm{~m}$, N35³2.995' E27¹0.187', leg. J. Kontschán, D. Murányi, 11.11.2012. HNHM/16664 1 ex., South Aegean, Naxos regional unit, Koronidha, stream above the village, $670 \mathrm{~m}, \mathrm{~N} 37^{\circ} 08.071^{\prime} \mathrm{E} 25^{\circ}$ 31.507', leg. J. Kontschán, D. Murányi, T. Szederjesi, 06.04.2013.

\section{Eisenia fetida (Savigny, 1826)}

Enterion fetidum Savigny, 1826: 182.

Eisenia foetida: Zicsi \& Michalis 1981: 254., Michalis 1982: 353., 1987: 63.

Eisenia fetida: Szederjesi \& Csuzdi 2012: 35.

Material examined. HNHM/16182 4 ex., South Aegean, Karpathos regional unit, Aperi, spring and stream in the village, $265 \mathrm{~m}$, N35 32.995' E27¹0.187', leg. J. Kontschán, D. Murányi, 11.11.2012. HNHM/16663 1 ex., South Aegean, Naxos regional unit, Koronidha, stream above the village, $670 \mathrm{~m}, \mathrm{~N} 37^{\circ} 08.071^{\prime} \mathrm{E} 25^{\circ}$ 31.507', leg. J. Kontschán, D. Murányi, T. Szederjesi, 06.04.2013.

\section{Eisenia oreophila Szederjesi \& Csuzdi, 2012}

Eisenia oreophila Szederjesi \& Csuzdi, 2012: 36.

Material examined. HNHM/16947 2 ex., Thessaly, Trikala peripheral unit, Lakmos Mts., Chaliki, open stream SW of the village, $1430 \mathrm{~m}$, N3940.267' E21 ${ }^{\circ} 09.176$ ', leg. T. Kovács, D. Murányi, 09.05.2014.

Remark. The specimens were found near the type locality.

\section{Eiseniella tetraedra (Savigny, 1826)}

Enterion tetraedrum Savigny, 1826: 184.

Eiseniella tetraedra tetraedra: Zicsi \& Michalis 1981: 242., 1993: 308., Michalis 1982: 524., 1995: 16.

Eiseniella tetraedra: Michalis 1987: 63., Szederjesi \& Csuzdi 2012: 37. 
Material examined. HNHM/16177 5 ex., South Aegean, Rhodes regional unit, Vati, roadside spring $\mathrm{E}$ of the village, $75 \mathrm{~m}$, N36 03.225' E27 ${ }^{\circ} 54.486$ ', leg. J. Kontschán, D. Murányi, 08.11.2012. HNHM/16621 1 ex., Crete, Heraklion regional unit, Agii Deka, small river and its gallery at Agios Tilos basilica, $160 \mathrm{~m}$, N3503.704' E2456.792', leg. J. Kontschán, D. Murányi, T. Szederjesi, 02.04.2013. HNHM/ 166231 ex., Crete, Lasithi regional unit, Thriptis Mts, Agios Ioannis, stream and its gallery E of the village, $460 \mathrm{~m}, \mathrm{~N} 35^{\circ} 03.615^{\prime} \mathrm{E} 25^{\circ} 51.938^{\prime}$, leg. J. Kontschán, D. Murányi, T. Szederjesi, 05.04. 2013. HNHM/16628 1 ex., South Aegean, Naxos regional unit, Mt. Zas, Filoti, vicinity of Zas

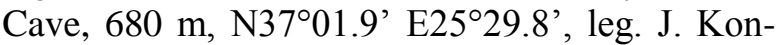
tschán, D. Murányi, T. Szederjesi, 07.04.2013. HNHM/16634 2 ex., Crete, Rethymno regional unit, Ida Mts., spring and its outlet at an archeogical site and taverna, nest of ants, $1380 \mathrm{~m}$, N35 $12.388^{\prime}$ E2450.044', leg. J. Kontschán, D. Murányi, T. Szederjesi, 02.04.2013. HNHM/ 166382 ex., South Aegean, Naxos regional unit, Ghalini, open stream at the village, $35 \mathrm{~m}$, N3706.888’ E25 $25.715^{\prime}$, leg. J. Kontschán, D. Murányi, T. Szederjesi, 06.04.2013. HNHM/ 166462 ex., Crete, Rethymno regional unit, Sisarcha, stream and its plane tree gallery $\mathrm{N}$ of the village, plane tree litter, $575 \mathrm{~m}, \mathrm{~N} 35^{\circ} 18.073^{\prime}$ E24⒌800', leg. J. Kontschán, D. Murányi, T. Szederjesi, 03.04.2013. HNHM/16655 2 ex., Crete, Chania regional unit, Skafi, stream and its plane tree gallery $\mathrm{S}$ of the village, mixed soil and plane tree litter, $370 \mathrm{~m}, \mathrm{~N} 35^{\circ} 18.806^{\prime}$ E23 ${ }^{\circ}$ 47.612', leg. J. Kontschán, D. Murányi, T. Szederjesi, 31.03.2013.

\section{Lumbricus rubellus Hoffmeister, 1843}

Lumbricus rubellus Hoffmeister, 1843: 187., Zicsi \& Michalis 1981: 255., Michalis 1975: 207., 1976: 163., 1982: 358., 1987: 63.

Material examined. HNHM/16069 1 ex., Thrace, Rhodope peripheral unit, Sapka Mts., Nea Sanda, open brook and pasture NE of the village, $790 \mathrm{~m}, \quad \mathrm{~N} 41^{\circ} 07.965^{\prime}$ E2554.052', leg. J. Kontschán, D. Murányi, T. Szederjesi, 26.05.
2012. HNHM/16659 1 ex., Crete, Chania regional unit, Kakopetros, stream and its plane tree gallery near the village, plane tree litter, $430 \mathrm{~m}$, N35²4.803' E23ํ⒌391', leg. J. Kontschán, D. Murányi, T. Szederjesi, 31.03.2013. HNHM/ 169481 ex., West Macedonia, Kozani peripheral unit, Neapoli, Aliakmonas River NE of the city, 555 m, N40¹9.976' E21 ${ }^{\circ} 24.678^{\prime}$, leg. T. Kovács, D. Murányi, 08.05.2014.

\section{Octodrilus complanatus (Dugès, 1828)}

Lumbricus complanatus Dugès, 1828: 289.

Octodrilus complanatus: Zicsi \& Michalis 1981: 256., 1993: 305., Michalis 1982: 357., 1987: 64., Szederjesi \& Csuzdi 2012: 38.

Octodrilus peleensis: Michalis, 1995: 15-20.

Material examined. HNHM/16092 3 ex., Thrace, Evros peripheral unit, Loutra Traianoupolis, river and thermal spring at the ruins, $15 \mathrm{~m}$, N4051.889’ E2601.881’, leg. J. Kontschán, D. Murányi, T. Szederjesi, 28.05.2012. HNHM/ 166161 ex., HNHM/16617 2 ex., Crete, Rethymno regional unit, Axos, spring $\mathrm{S}$ of the village, 590 m, N35 ${ }^{\circ} 17.934^{\prime}$ E24 $50.485^{\prime}$, leg. J. Kontschán, D. Murányi, T. Szederjesi, 02.04. 2013. HNHM/16618 3 ex., Crete, Heraklion regional unit, Agii Deka, small river and its gallery at Agios Tilos basilica, $160 \mathrm{~m}, \quad \mathrm{~N} 35^{\circ} 03.704^{\prime}$ E2456.792', leg. J. Kontschán, D. Murányi, T. Szederjesi, 02.04.2013. HNHM/16645 1 ex., Crete, Rethymno regional unit, Sisarcha, stream and its plane tree gallery $\mathrm{N}$ of the village, plane tree litter, $575 \mathrm{~m}, \mathrm{~N} 35^{\circ} 18.073^{\prime} \mathrm{E} 24^{\circ} 54.800^{\prime}$, leg. J. Kontschán, D. Murányi, T. Szederjesi, 03.04. 2013. HNHM/16650 1 ex., HNHM/16652 6 ex., Crete, Rethymno regional unit, Moni Veni, springs at the monastery, soil, $595 \mathrm{~m}$, N35⒗228' E2436.377', leg. J. Kontschán, D. Murányi, T. Szederjesi, 01.04.2013. HNHM/ 166531 ex., Crete, Rethymno regional unit, Apostoli, stream and its plane tree gallery $\mathrm{N}$ of the village, $320 \mathrm{~m}, \mathrm{~N} 35^{\circ} 16.211^{\prime} \mathrm{E} 24^{\circ} 36.821^{\prime}$, leg. J. Kontschán, D. Murányi, T. Szederjesi, 01.04. 2013. HNHM/16654 1 ex., Crete, Rethymno regional unit, Goulediana, olive grove with oak stands at the village, $440 \mathrm{~m}, \mathrm{~N} 35^{\circ} 17.206^{\prime}$ 
E24²9.949’, leg. J. Kontschán, D. Murányi, T. Szederjesi, 01.04.2013. HNHM/16665 1 ex., Crete, Lasithi regional unit, Dikti Mts., Katharo, rocky evergreen oak forest $\mathrm{E}$ of the settlement, mixed soil and leaf litter, $1070 \mathrm{~m}, \mathrm{~N} 35^{\circ} 09.242^{\prime}$ E25³5.185', leg. J. Kontschán, D. Murányi, T. Szederjesi, 03.04.2013.

\section{Octodrilus croaticus (Rosa, 1895)}

Allolobophora lissaensis var. croatica Rosa, 1895: 5.

Octodrilus croaticus: Zicsi \& Michalis 1981: 257., 1993: 305., Michalis 1982: 358., Szederjesi \& Csuzdi 2012: 39.

Material examined. HNHM/16944 2 ex., Thessaly, Trikala peripheral unit, Lakmos Mts., Chaliki, rocky grassland $\mathrm{W}$ of the village, 1715 m, N3940.516' E2 $1^{\circ} 08.583$ ', leg. T. Kovács, D. Murányi, 09.05.2014. HNHM/16945 3 ex., Thessaly, Trikala peripheral unit, Lakmos Mts., Chaliki, stream below Verliga Waterfall, $1935 \mathrm{~m}$, N3940.674' E2107.818', leg. T. Kovács, D. Murányi, 09.05.2014.

\section{Octodrilus transpadanus (Rosa, 1884)}

Allolobophora transpadana Rosa, 1884: 45.

Octodrilus transpadanus: Zicsi \& Michalis 1981: 257., 1993: 305., Michalis 1982: 358., 1995: 16., Szederjesi \& Csuzdi 2012: 39.

Octodrilus transpadanum: Michalis 1987: 64.

Material examined. HNHM/16076 4 ex., HNHM/16079 4 ex., Thrace, Evros peripheral unit, Lesitse Mts., Loutros, stream and its gallery $\mathrm{N}$ of the village, $55 \mathrm{~m}, \mathrm{~N} 40^{\circ} 55.485^{\prime} \mathrm{E} 26^{\circ} 03.673^{\prime}$, leg. J. Kontschán, D. Murányi, T. Szederjesi, 27.05.2012. HNHM/16086 3 ex., Thrace, Rhodope peripheral unit, Tsifliki, slow stream SW of the village, $35 \mathrm{~m}, \mathrm{~N} 41^{\circ} 03.085^{\prime} \mathrm{E} 25^{\circ} 37.569^{\prime}$, leg. J. Kontschán, D. Murányi, T. Szederjesi, 26.05. 2012.

\section{Family Acanthodrilidae Claus, 1880}

\section{Microscolex dubius (Fletcher, 1887)}

Eudrilus dubius Fletcher, 1887: 378.
Microscolex dubius: Cognetti 1913: 1., Michalis 1975a: 202., 1976: 154., 1985: 348., 1987: 61., Szederjesi \& Csuzdi 2012: 40.

Microscolex dibius: Michalis 1975b: 189.

Material examined. HNHM AF/5565 1 ex., South Aegean, Naxos regional unit, Mt. Zas, Filoti, vicinity of Zas Cave, $680 \mathrm{~m}, \mathrm{~N} 37^{\circ} 01.9^{\prime}$ E25'29.8', leg. J. Kontschán, D. Murányi, T. Szederjesi, 07.04.2013. HNHM AF/5567 1 ex., South Aegean, Naxos regional unit, Apiranthos, olive orchard $\mathrm{E}$ of the village, $525 \mathrm{~m}$, $\mathrm{N} 37^{\circ} 04.408^{\prime} \mathrm{E} 25^{\circ} 31.842^{\prime}$, leg. J. Kontschán, D. Murányi, T. Szederjesi, 07.04.2013. HNHM AF/5568 1 ex., Crete, Heraklion regional unit, Krasi, spring system in the village, $610 \mathrm{~m}$, $\mathrm{N} 35^{\circ} 14.010^{\prime} \mathrm{E} 25^{\circ} 28.154$ ', leg. J. Kontschán, D. Murányi, T. Szederjesi, 03.04.2013. HNHM AF/5569 5 ex., Crete, Chania regional unit, Sougia, seashore tamarisk stands at the village, 0 m, N35⒕917' E23ํ⒏706', leg. J. Kontschán, D. Murányi, T. Szederjesi, 31.03.2013.

\section{Microscolex phosphoreus Dugès, 1837}

Lumbricus phosphoreus Dugès, 1837: 17.

Microscolex phosphoreus: Michalis 1975a: 202., 1976: 154.

Material examined. HNHM AF/5566 1 ex., South Aegean, Naxos regional unit, Mt. Zas, Filoti, vicinity of Zas Cave, $680 \mathrm{~m}, \mathrm{~N} 37^{\circ} 01.9^{\prime}$ E25²9.8', leg. J. Kontschán, D. Murányi, T. Szederjesi, 07.04.2013.

\section{Family Megascolecidae Rosa, 1891}

\section{Pontodrilus litoralis (Grube, 1855)}

Lumbricus litoralis Grube, 1855: 127.

Pontodrilus litoralis: Blakemore 2007: 4.

Material examined. HNHM AF/5562 6 ex., Thrace, Evros peripheral unit, Dikelle, rocky seashore SE of the village, $0 \mathrm{~m}, \mathrm{~N} 40^{\circ} 50.910^{\prime}$ E25 42.440', leg. J. Kontschán, D. Murányi, T. Szederjesi, 27.05.2012.

Remarks. Pontodrilus litoralis is a cosmopolitan shoreline species, distributed from the 
Mediterranean see-shores to India (Narayanan et al. 2014) the Caribbean (Csuzdi \& Pavlíček 2009) and the pacific regions (Blakemore 2007). This is the first record from Greece.

\section{Family Ocnerodrilidae Beddard, 1891}

\section{Ocnerodrilus occidentalis Eisen, 1878}

Ocnerodrilus occidentalis Eisen, 1878: 10., Blakemore 2008: 149.

Material examined. HNHM AF/5561 1 ex., South Aegean, Rhodes regional unit, Aghios Nektarios, pine forest $\mathrm{E}$ of the monastery, $145 \mathrm{~m}$, N36 15.943 ' E2804.822', leg. J. Kontschán, D. Murányi 14.11.2012.

Remark. This species is new to the fauna of Greece.

\section{DISCUSSION}

Out of the 22 earthworm species and subspecies found, three proved to be new to the fauna of Greece. Dendrobaena pentheri was described from Turkey (Rosa 1905). It has later been reported from Azerbaijan (as Helodrilus (Eisenia) schemachaensis Michaelsen, 1910) Gerogia, Armenia (Kvavadze 1985) and, recently from Cyprus (Pavlíček \& Csuzdi 2006, Pavlíček et al. 2010). Here we proved that D. aegea described from Rhodes is a synonym of Dendrobaena pentheri and in this sense $D$. pentheri is a new species name in the list of the earthworm species of Greece.

Pontodrilus litoralis is of uncertain origin (Blakemore 2007) and found all over the world on the shorelines of warmer oceans and seas. Our specimens were found in seashore debris on the Aegean coast.

The South American Ocnerodrilus occidentalis is widespread as well in tropical and Mediterranean regions, and can be found even in cooler climates, e.g. in Hungary in greenhouses (Csuzdi et al. 2007).

Together with these new records, the number of earthworm species recorded for Greece is 59 .
Acknowledgements - My thanks are due to Dávid Murányi (HNHM Budapest) and Jenő Kontschán (PPI Budapest) for their kind assistance during the field work and collecting earthworms in Rhodes and Karpathos. This research was supported by a grant from OTKA (No. 100369).

\section{REFERENCES}

BLAKEMORE, R.J. (2007): Origin and means of dispersal of cosmopolitan Pontodrilus litoralis (Oligochaeta: Megascolecidae). European Journal of Soil Biology, 43: S3-S8. doi: 10.1016/j.ejsobi.2007.08.041

BLAKEMORE, R.J. (2008): Cosmopolitan Earthworms ( $3^{\text {rd }}$ Edition). VermEcology, Yokohama, Japan, pp. 757.

CognetTi, L. (1906): Nuovi dati sui Lumbricidi dell' Europa orientale. Bollettino dei Musei di zoologia ed anatomia comparata della $R$. Università di Torino, 21(257): 1-18.

CognetTi, L. (1913): Escursioni zoologiche del Dr. E. Festa nell'Isola di Rodi V. Oligocheti. Bollettino dei Musei di zoologia ed anatomia comparata della R. Università di Torino, 28(74): 1-6.

ČERNOSVITOV, L. (1934): Sur les Oligochètes terricoles de Crète. Sbornik Zoologického Oddeleni Narodniho Musea v Praze, 1(4): 17-20.

CsUZDI, Cs. (2012): Earthworm species, a searchable database. Opuscula Zoologica Budapest, 43(1): 9799.

Csuzdi, Cs., PAvlíčEK, T. \& Nevo, E. (2007): Is Dichogaster bolaui (Michaelsen, 1891) the first domicole earthworm species? European Journal of Soil Biology, 44: 198-201.

doi: $\underline{10.1016 / j . e j s o b i .2007 .05 .003}$

Csuzdi, Cs. \& PAVLíčEK, T. (2009): New records of earthworms from Guadeloupe with description of a new species (Oligochaeta; Glossoscolecidae, Acanthodrilidae, Megascolecidae and Eudrilidae). Opuscula Zooogica, Budapest, 40(1): 9-15.

DUGÈs, A. (1828): Recherche sur la circulation, la respiration, et la reproduction des Annélides sétigéres abranches. Annales des Sciences Naturelles Paris, 15: 284-336.

DuGĖS, A. (1837): Nouvelles observations sur la zoologie et l'anatomie des Annélides sétigéres abranches. Annales des Sciences Naturelles Paris ser. 2 Zoologie, 8: 15-35. 
EISEN, G. (1873): Om Skandinaviens Lumbricider. Öfversigt af Kongliga Vetenskaps-Akademiens Förhandligar, 30(8): 43-56.

EISEN, G. (1878): On the Anatomy of Ocnerodrilus. Nova Acta Regiae Societatis Scientiarum Upsaliensis, 10(4): 1-12.

FletCHER, J.J. (1887): Notes on Australian Earthworms. Part III. Proceedings of the Linnean Society of NSW, (2)2: 377-402.

GRUBE, E. (1855): Beschreibungen neuer oder wenig bekannter Anneliden. Archiv für Naturgeschichte, 27: $81-136$

HOFFMEISTER, W. (1843): Beitrag zur Kenntnis deutcher Landanneliden. Archiv für Naturgeschichte, 91: 183-198.

KARAMAN, S. (1972): Beitrag zur Kenntnis der Lumbricidenfauna von Griechenland. Fragmenta Balcanica Skopje, 9(11): 109-115.

KVAVADZE E.S. (1985): The earthworms (Lumbricidae) of the Caucasus. Metsniereba, Tbilisi, pp. 237.

Michaelsen, W. (1890): Die Lumbriciden Norddeutschlands. Jahrbuch der Hamburgischen Wisseschaftlichen Anstalten, 7: 1-19.

Michaelsen, W. (1891): Oligochaeten des Naturhistorischen Museums in Hamburg IV. Jahrbuch der Hamburgischen Wissenschaftlichen Anstalten, 8: $1-42$.

MichaElsen, W. (1902): Neue Oligochaeten und neue Fundorte alt-bekannter. Mitteilungen aus dem $\mathrm{Na}$ turhistorischen Museum in Hamburg, 19: 3-53.

MiChAELSEN, W. (1903): Die geographische Verbreitung der Oligochaeten. Friedländer \& Sohn, Berlin pp. 186.

MichAELSEN, W. (1914): Ein neuer Regenwurm aus Griechenland. Verhandlungen der Zoologisch-Botanischen Gesellschaft in Wien, 8: 8-9.

MichaElsen, W. (1928): Beiträge zur Kenntnis der Fauna Griechenlands, namentlich der ägäischen Inseln. Sitzungsberichte der Kaiserlichen Akademie der Wissenschaften in Wien, 137: 289-290.
Michalis, K. (1975a): Neue Angaben zur Fauna der Oligochaeten von Mazedonien. Scientific Annals, Faculty of Physics and Mathematics, University of Thessaloniki, 15: 201-211.

MichALIS, K. (1975b): Die Oligochaeten der Insel Kos. Scientific Annals, Faculty of Physics and Mathematics, University of Thessaloniki, 15: 187-200.

Michalis, K. (1976): Beitrag zur Kenntnis der Oligochaetenfauna Thessaliens. Scientific Annals, Faculty of Physics and Mathematics, University of Thessaloniki, 16: 153-168.

MicHALIS, K. (1977): Die Oligochaetenfauna der Halbinsel von Athos (Chalkidiki). Scientific Annals, Faculty of Physics and Mathematics, University of Thessaloniki, 17: 285-298.

MiCHALIS, K. (1982): Katalog der Oligochaetenfauna Griechenlands. Biologia Gallo-Hellenica, 9: 343 362.

MicHALIS, K. (1983): New biotopes of the species Allolobophora dofleini (Ude, 1922) and the subspecies Bimastus antiquus bouchei (Zicsi-Michalis, 1981) (Oligochaeta-Lumbricidae). Ecologia Mediterranea, 9: 57-61.

MichALIS, K. (1987): Contribution to the systematic and ecological study of the earthworms (Oligochaeta, Lumbricidae) of the Western Thrace. Arquivos do Museu Bocage, 1(5): 59-69.

MichaLIS, K. (1995): Oligochaeten - Funde aus Thessalien (Griechenland) nebst Beschreibung der Art Octodrilus peleensis sp. nov. Bios (Macedonia, Greece), 3: 15-20.

NARAYANAN S. P., SATHRUMithra, S., Kuriakose, D., Christopher, D., ThOMAS A. P. \& JULKA, J. M. (2014): Second record of Pontodrilus litoralis (Grube, 1855) (Annelida: Oligochaeta) from Kerala state, India. Journal of Global Biosciences, 3(2): 473-476.

OMODEO, P. (1955): Lombrichi cavernicoli di Grecia e Turchia raccolti dal Dr. K. Lindberg. Annuario dell Istituto e Museo di Zoologia della Università di Napoli, 7: 1-16.

PAVlíČEK, T. \& CSUZDI, Cs. (2006): Species richness and zoogeographicaffinities of earthworms in Cyprus. European Journal of Soil Biology, 42S: 111-116. doi: 10.1016/j.ejsobi.2006.09.001 
PAVlíČEK, T., CSUZDI, CS., MisiRlioĞLU, M. \& VILENKIN, B. (2010): Faunistic similarity and endemism of earthworms in east Mediterranean region. Biodiversity and Conservation, 19: 1989-2001. doi: $\underline{10.1007 / \mathrm{s} 10531-010-9821-1}$

RAW, F. (1959): Estimating earthworm population by using formalin. Nature, 184: 1661-1662. doi: $10.1038 / 1841661 \mathrm{a} 0$

RosA, D. (1884): Lumbricidi del Pieomte. Unione Tipografico-Editrice, Torino, pp. 54.

RosA, D. (1886): Note sui lombrici del Veneto. Atti del Reale Istituto Veneto di Scienze, 4: 673-687.

RosA, D. (1893): Viaggio del Dr. E. Festa in Palestina, nel Libano e regioni vicine. - II. Lumbricidi. Bollettino dei Musei di Zoologia ed Anatomia comparata della R. Università di Torino, 8(160): 1-14.

RosA, D. (1895): Nuovi lombrichi dell'Europa orienttale. Bollettino dei Musei di Zoologia ed Anatomia comparata della $R$. Università di Torino, 10(21): $1-8$.

RosA, D. (1905): Terricolen. In: Ergebnisse einer Naturwissenschaftlichen Reise zum Erdschias Dag. Annalen des K.K. Naturhistorischen Hofmuseums Wien, 20: 104-106.

SAVIGNY, J.C. (1826): In G. CuVIER: Analyse des Travaux de l'Académie royale des Sciences, pendant l'année 1821, partie physique. Mémoires de l'Académie des Sciences de l'Institut de France Paris, 5: 176-184.
ŠAPKAREV， J. (1972): Beiträge zur Kenntnis der Lumbricidenfauna Griechenlands. Macedonian Academy of Sciences and Arts, 4: 31-36.

TZELEPIS, N.D. (1943): Symvoli is tin meletin ton Oligochaeton tis Ellados. Athen, pp. 60.

SZEDERJESI, T. \& CsUZDI, Cs. (2012): New and little known earthworm species from Greece (Oligochaeta: Lumbricidae, Acanthodrilidae). Zootaxa, 3304: $25-42$.

SzEderJesi, T., PAVlíčEK, T., CoşKun, Y. \& CsuZdi, Cs. (2014): New earthworm records from Turkey, with description of three new species (Oligochaeta: Lumbricidae). Zootaxa, 3764(5): 555-570. doi: $\underline{10.11646 / \text { zootaxa.3764.5.4 }}$

ZICSI, A. (1973): Regenwürmer (Oligochaeta: Lumbricidae) aus Griechenland. Opuscula Zoologica Budapest, 12: 99-103.

ZICSI, A. (1974): Ein neue Dendrobaena-Art (Oligochaeta: Lumbricidae) aus Griechenland. Acta zoologica hungarica, 20: 449-451.

ZICSI, A. \& MiCHALIS, K. (1981): Übersicht der Regenwurm-fauna Griechenlands (Oligochaeta: Lumbricidae). Acta Zoologica Hungarica, 27: 239-264.

ZICSI, A. \& Michalis, K. (1993): Zwei neue Dendrobaena-Arten aus Grichenland (Oligochaeta: Lumbricidae). Acta Zoologica Hungarica, 39: 301310 . 